\title{
Some General Results and Prospects for the Development of Clinical Immunology of the XIX - XXI Centuries
}

ISSN: 2637-773X

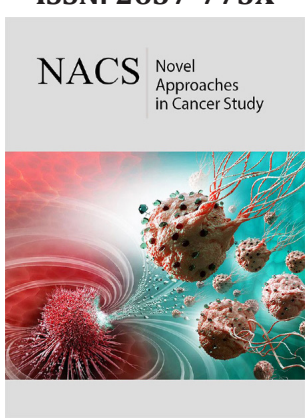

*Corresponding author: Vladimir M Zemskov, AV Vishnevsky National Medical Research Center of Surgery, Moscow, 115998, Bolshaya Serpukhovskaya 27, Russia

Submission: 柴 November 08, 2019

Published: 眥 November 22, 2019

Volume 3 - Issue 5

How to cite this article: Zemskov VM Zemskov A. Some General Results and Prospects for the Development of Clinical Immunology of the XIX - XXI Centuries. Nov Appro in Can Study. 3(5). NACS.000575.2019.

DOI: 10.31031/NACS.2019.03.000575

Copyright@ Vladimir M Zemskov, This article is distributed under the terms of the Creative Commons Attribution 4.0 International License, which permits unrestricted use and redistribution provided that the original author and source are credited.

\author{
Zemskov VM${ }^{1,2 *}$ and Zemskov $\mathrm{AM}^{3}$ \\ ${ }^{1} \mathrm{AV}$ Vishnevsky National Medical Research Center of Surgery, Russia \\ ${ }^{2}$ Department of Research and Development at Face control, USA \\ ${ }^{3}$ Department of Microbiology of Burdenko, Russia
}

\section{Introduction}

Since the student audience is generally very limited and the materials presented in the textbooks are not widely available to a wide circle of members of the scientific community, the authors decided to present for attention some purely general fundamental problems of clinical immunology that are presented in our textbooks for students of medical universities. The proposed materials are borrowed from our student textbooks [1,2].

Clinical immunology is a branch of clinical medicine that studies the pathogenesis, diagnosis, treatment, and prevention of diseases, which are based on disorders of the immune system functions or conditions that cause severe impaired immune reactivity that require special treatment (correction).

The objectives of clinical immunology:

1) Diagnosis and treatment of congenital immune deficiency (primary immunodeficiencies).

2) Detection and treatment of secondary (acquired) immunodeficiencies.

3) Diagnosis and treatment of somatic immunopathological disorders.

4) Diagnosis and treatment of allergies.

5) Diagnosis and treatment of lymphoproliferative diseases.

6) Diagnosis and treatment of autoimmune diseases.

7) Immunogenetic selection of a pair of donor-recipient, the creation of immune chimeras.

8) Diagnosis of immune disorders in cancer pathology and their therapy.

9) Detection of immune defects and their targeted elimination.

10) Diagnosis and treatment of immune reproductive disorders. These include pregnancy, lactation, infertility, menopause.

11) Development of innovative technological methods for the fine diagnosis of

12) cellular, tissue and organ disorders, immune vectors for targeted drug transport.

Over the short history of scientific immunology, approximately 100 years, has led to the realization of major practical medical problems.

A. The creation of vaccines and vaccinations against smallpox, rabies, diphtheria, anthrax, pertussis, polio, tetanus, measles, gas gangrene, including drinking especially dangerous infections. By 1978, smallpox was successfully eradicated. 
B. Blood transplantation with determination of $\mathrm{ABO}$ and other blood antigens, which is extremely important for blood transfusion, obstetrics, forensic medicine, medical genetics, etc.

C. The treatment of rhesus hemolytic disease of the newborn.

D. The discovery of immune tolerance, drug immunosuppression, which made possible the transplantation of foreign organs with $80-90 \%$ of their engraftment.

E. Optimization of accurate, including automated diagnosis of infectious and non-infectious diseases, allergies, autoimmune pathology, immunodeficiencies, leukemia, Rh incompatibility.

The fundamental significance of immunology lies in the basic axioms that have changed many times over time.

\section{Axioms of the late XIX - early XX century}

a. The immune system is basically a phagocyte system.

b. Cells of the immune system cannot respond to antigens of their own body.

c. Immunity - mainly resistance to infectious diseases.

d. The main material substrate of life and heredity are proteins.

\section{Axioms of the second quarter of the twentieth century}

a. The immune system is mainly represented by the reticuloendothelial system.

b. The antigen installs new information into the cells of the immune system.

c. Under certain conditions, cells of the body's immune system can react against their own antigens.

d. Immunity is the ability to respond not only against foreign microbes, but al-so any foreign genetic information.

e. One gene determines the synthesis of only one protein.

f. The genetic structure of heredity is DNA.

g. Transportation of genetic information occurs along the line: DNA $\rightarrow$ RNA $\rightarrow$ protein.

\section{Axioms of the third quarter of the twentieth century}

a. The immune system consists mainly of lymphoid cells and their derivatives. Secondary "extra" cells (leukocytes, macrophages, monocytes, mast cells, eosinophils, etc.) are secondary.

b. All the extensive possible immune information against all possible antigens, including artificial ones, pre-exists in the genome of immune cells. Antigen does not introduce new information into cells.

c. The immune system consists of central and peripheral organs.

d. One specific antibody-forming cell can synthesize only one antibody of narrow specificity. e. The immune response is carried out during the cooperation or suppression of various cells of the immune system.

f. The synthesis of a single protein can be determined by several genes.

g. The transfer of genetic information can occur along the lines of DNA $\rightarrow$ RNA or RNA $\rightarrow$ DNA $\rightarrow$ protein.

\section{Axioms of the last quarter of the twentieth century}

a. Any chronic and severe acute pathological processes are accompanied by disorders of the immune system, contributing to the aggravation of the disease, its relapse, complications.

b. Without the elimination of immune active disorders, the treatment of severe and chronic diseases cannot be considered qualified.

c. Almost any drug and non-drug interventions (physiotherapeutic procedures, acupuncture, plasmapheresis, etc.) turn out to be "immunotropic," that is, they stimulate or suppress the immune system.

d. The development of immune responses is inextricably linked with the function of the endocrine, nervous, excretory, circulatory and other body systems.

e. Most likely, the immune system is not autonomous, but represents the most important link in the more general integral system of the body, mentioned in paragraph 4 .

f. Immune disorders are typical, accompanied by metabolic changes in the body.

g. Immunocorrectors are endowed not only with a profile, but also with a general metabolic effect.

\section{Axioms of the beginning of the $21^{\text {st }}$ century}

a. Red blood cells, erythropoietin, platelets, neutrophils, basophils and eosinophils, nerve, muscle and other types of cells, metabolic disorders, modification of oxidative and antioxidant systems, and other non-lymphoid mechanisms participate in the development of specific immune reactions.

b. In the formation of the same type of pathological processes, the immune system can develop alternative (multidirectional) reactions.

c. The induction of immune mechanisms, the effectiveness of drugs, modulators, and non-drug effects are affected not only by the genes of the HLA system, but also by the carriage of antigens of the $\mathrm{ABO}$ system, Rh factor, haptoglobin, etc.

d. Immune reactions, destroying microorganisms, malignant, altered somatic cells, release biologically active substances that regulate the body's immune homeostasis.

e. With the development of various diseases, the immune mechanisms enter in-to typical interactions with each other, with hematological and metabolic structures (intra-, inter- and extrasystemic associations), the severity of which de-pends on the 
nature, stage of the pathological process, type of treatment and other reasons.

f. The effectiveness of directional correction of altered defense reactions is determined by the degree of reproduction of naturally evolutionarily fixed mechanisms of restoration of impaired immune homeostasis.

According to the famous Russian immunologist Academician Petrov R and other researchers, immunology in the coming years should contribute to solving the following problems.

A. Prevention of not yet defeated infections of humans and farm animals.

B. The search for approaches to stimulating immunity against new artificially synthesized aggressive agents of the external environment (industry, chemicalization of the economy, etc.).

C. The creation of effective methods for the recovery of secondary immunodeficiencies in chronic infectious and noninfectious diseases, selective modulators of specific immune units.

D. Prevention and treatment of autoimmune, including rheumatic, diseases by improving immunosuppressive therapy.

E. Improving the effectiveness of immunoprophylaxis, immunodiagnosis and immunothery of tumors, including immunoproliferative diseases, lymphomas, etc.

F. Activation of basic research on the microenvironment of tumor cells, inhibitors of "immune points" and the reduction of chemotherapy toxicity, the directed transport of killer cell effectors into tumors and the reprogramming of cancer cells to harmless ones. Carrying out developments in tumor pathology, built on new physical principles and biophysical laws.

G. Prevention and treatment of allergies.

H. Reducing immune complications in obstetric practice and infant mortality caused by the mother-fetus immune conflict, primary immunodeficiencies.

I. Development of new methods for predicting rejection in the induction of immune tolerance, in organ and tissue transplantation.

J. Compensation of damage to immune mechanisms caused by external adverse environmental factors.

Creation of new highly specific and supersensitive immune methods for detecting trace amounts of harmful organic and biological substances, micro-chips for diagnosing pathological conditions outside the body, new therapeutic immune preparations, including non-invasive therapy.

\section{References}

1. Zemskov AM, Zemskov VM, Karaulov AV (2008) Clinical immunology. College textbook. GEOTAR-Media Publ, Moscow, Russia:

2. Zemskov AM, Esaulenko IE, Chereshnev VA, Zemskov VM (2016) Immunology. Electronic Textbook for students of medical universities and faculties with applications, GEOTAR-Media Publ, Moscow, Russia. 